ECOLOGY

\section{Longing for home undid cave bears}

When looking for a spot to hibernate, ancient cave bears stuck with family.

Cave bears (Ursus spelaeus) went extinct 24,000 years ago, whereas the related brown bears (Ursus arctos) still thrive. To explain these differing fates, a team led by Gloria Fortes and Axel Barlow at the University of Potsdam, Germany, obtained mitochondrial genomes of 31 cave bears and 15 ancient brown bears from caves in Spain. Cave bears from the same caves tended to share mitochondrial DNA, which is inherited maternally, whereas brown-bear caves contained multiple maternal lines.

These patterns suggest that cave bears nearly always hibernated in their native caves, which may have contributed to their demise in the face of competition from other bears, the scientists say.

Mol. Ecol. http://doi.org/bpgr (2016)

\section{ECONOMICS}

\section{Satellites map world poverty}

Computer scientists have used satellite imagery and machine-learning techniques to make detailed maps of regions where poverty is common.

Neal Jean and his colleagues at Stanford University in California focused on Nigeria, Tanzania, Uganda, Malawi and Rwanda and combined various data sets, including daytime images that identify features such as paved roads and metal roofs, to estimate local household consumption

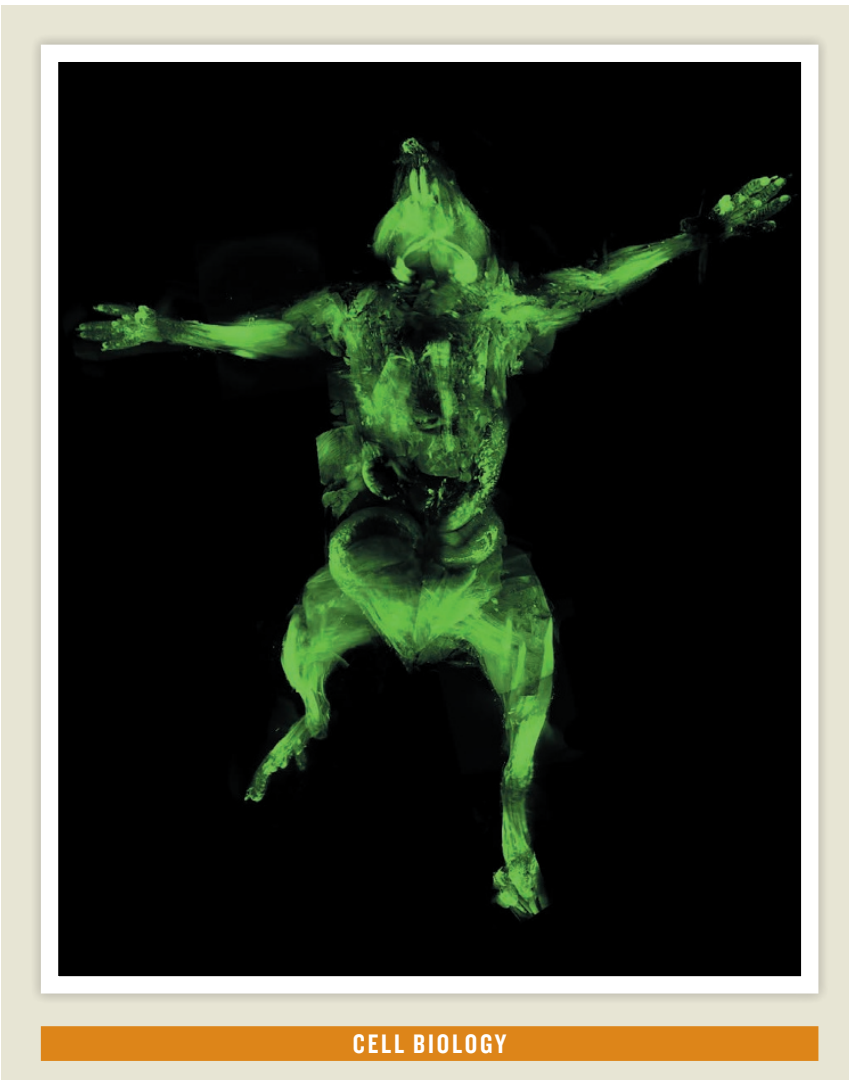

\section{See-through rodents}

An imaging technique lets scientists peer through the skin of a whole mouse or rat to examine its organs after death.

Ali Ertürk of the Ludwig Maximilians University of Munich in Germany and his colleagues created a technique called ultimate DISCO (uDISCO), which removes pigments and lipids from the tissues of dead animals using an organic solvent. This leaves the organs and skin intact but transparent, while preserving genetically encoded fluorescent proteins. The method revealed the nervous system of a mouse in stark detail.

uDISCO also shrinks bodies by up to $65 \%$, making it possible to image whole animals using light-sheet microscopy, which excels at imaging smaller samples.

Nature Methods http://dx.doi.org/10.1038/nmeth.3964 (2016) and income. When identifying areas where incomes are below the international poverty line of US $\$ 1.90$ per person per day, the team's algorithm outperforms night-light maps (an alternative but limited indicator of economic activity). It also probes hardto-reach areas, including regions not accessed by household surveys - such as those conducted by the World Bank - which are costly and infrequently conducted.

The method could prove useful for targeting social programmes and determining Science 353, 790-794 (2016) when and where they fail.

\section{GENOMICS \\ Medicine less precise for some}

A lack of ethnic diversity in people whose genomes have been sequenced is complicating precision medicine for people with non-European ancestry.

David Goldstein of Columbia University in New York City and Slavé Petrovski of the Royal Melbourne Hospital in Australia examined data from the Exome Aggregation Consortium (ExAC), which contains sequences from 60,252 people, $60.9 \%$ of whom have European ancestry. When they compared genetic variants from a cohort of 5,094 people with variants found in the ExAC and other data sets, the comparisons yielded a shorter list of potentially causative variants in people with European ancestry (6.6, on average) than in people with non-European ancestry (9.9-12.7 candidate variants, depending on ethnicity).

Precision medicine is much more precise for people with European than nonEuropean ancestry owing to undersampling of nonEuropean populations, the authors write.

Genome Biol. http://doi.org/bphp (2016)

\section{PARTICLE PHYSICS}

\section{Neutrino search closes in}

Scientists are getting closer to discovering whether neutrinos and antineutrinos are in fact the same particles - known as Majorana neutrinos.

The theory, proposed by Italian physicist Ettore Majorana in the 1930s, could 